\title{
Electronic K-x-ray transitions in light muonic atoms
}

\author{
Takeshi Mukoyama ${ }^{a}$ \\ Institute for Nuclear Research of the Hungarian Academy of Sciences (ATOMKI), Bem tér 18/c, H-4026 Debrecen, \\ Hungary
}

Received 14 November 2018 / Received in final form 17 January 2019

Published online 9 April 2019

(c) The Author(s) 2019. This article is published with open access at Springerlink.com

\begin{abstract}
Energies and radiative transition rates of electronic K x-rays in light muonic atoms have been calculated for various muonic states. The energy eigenvalues and wave functions of atomic electrons and the muon are obtained by solving the equation for the electron-muon system self-consistently. The $x$-ray energies are expressed as difference from the energy of the ordinary x-rays with atomic number $(Z-1)$ and the radiative rates are obtained as the ratio to the values for $(Z-1)$ atom. The energy shifts and relative radiative rates are studied as a function of $n$ and $l$ of the muonic state. The effect of additional vacancies in the electronic $2 p$ orbital on the $\mathrm{K}$-x-ray energies and radiative transition rates is discussed.
\end{abstract}

\section{Introduction}

When negative muons are slowing down and stop in matters, they are usually captured by atoms of the target materials and muonic atoms are formed. In general, the muon is captured into one of highly excited states and then cascades down to lower-energy states through emission of $\mathrm{x}$-rays and Auger electrons. During the cascade process the interplay between the muon and atomic electrons is important. After the muon reaches the $1 s$ ground state, it either decays into an electron-neutrino pair or is absorbed by the nucleus.

In addition to the muonic x-rays due to the transitions of the muon between atomic orbitals, three types of x-rays originated from electron transitions appear in the prompt and delayed spectra. The ordinary x-rays of the target elements excited by slowing-down muons are measured as both prompt and delayed signals. After the muon is captured by the atomic nucleus, x-rays from the daughter element with atomic number less by one are emitted as the delayed spectra. It is also possible to observe x-ray lines in the prompt spectrum, which are emitted when the muon stays in an atomic orbital. The x-rays in the last case, called electronic x-rays from muonic atoms, can give information of the electronic structure of the muonic atom and are the subject of the present work.

There have been reported extensive theoretical and experimental works on the energies and intensities of muonic $\mathrm{x}$-rays for various elements [1]. Recently the

\footnotetext{
* Contribution to the Topical Issue "Many Particle Spectroscopy of Atoms, Molecules, Clusters and Surfaces (2018)", edited by Károly Tőkési, Béla Paripás, Gábor Pszota, and Andrey V. Solov'yov.

a e-mail: hakutei@rhythm.ocn.ne.jp
}

muonic x-rays have been applied for elemental analysis [2]. On the other hand, studies on electronic x-rays are rather scarce and limited to the energy shifts of the x-ray lines in heavy atoms.

For electronic x-rays from muonic atoms, energy shifts have been observed [3-6] and it is also expected that the $\mathrm{x}$-ray emission rates or relative intensities of $\mathrm{x}$-ray transitions are changed. These results are attributed to two effects, i.e. screening of the nuclear charge by the muon and existence of vacancies created in the electronic shells during muonic cascade. In the former case, the $\mathrm{x}$-ray energies become lower than those in the ordinary atom due to the increase in screening and the radiative transition rates are slightly smaller. On the other hand, the existence of inner-shell electron vacancies decreases the screening effect and increases the $\mathrm{x}$-ray energies. The $\mathrm{x}$-ray emission rates depend on the vacancy distribution, but are strongly reduced when the vacancies are located at the initial shell involved in the x-ray transition.

Electron screening in muonic atoms has been estimated with various atomic models [7-9] and used to compare with the experimental values for the transition energies of muonic x-rays [10]. The calculations on energies of electronic $\mathrm{K}$ x-rays in muonic atoms have been reported for muons in various atomic orbitals and for different heavy elements $[5,11,12]$. The $\mathrm{x}$-ray energies are usually expressed as the energy shifts relative to the diagram line of the ordinary $(Z-1)$ atom, where $Z$ is the atomic number of the target element. All these studies were made for the elements with $Z \geq 50$ and no calculation has been done for light elements.

In the present work, the energy shifts and relative emission rates of the electronic $\mathrm{K}$ x-rays in muonic atoms are calculated for light elements. The atomic electrons and the muon are treated self-consistently within the framework of the nonrelativistic Hartree-Fock-Slater (HFS) model [13]. 
Table 1. Comparison of energy eigenvalues $(\mathrm{eV})$ for $\mu^{-} \mathrm{Al}^{+}$atom with those of $\mathrm{Mg}$ and $\mathrm{Al}$ atoms.

\begin{tabular}{llllllll}
\hline & & \multicolumn{5}{c}{ Muonic state } & \\
\cline { 3 - 7 } & $\mathrm{Mg}$ & $1 s$ & $5 s$ & $10 s$ & $14 s$ & $20 s$ & $\mathrm{Al}$ \\
\hline $1 s$ & -1291 & -1291 & -1296 & -1340 & -1413 & -1506 & -1546 \\
$2 s$ & -89.1 & -89.1 & -89.4 & -90.8 & -95.2 & -96.4 & -118.5 \\
$2 p$ & -56.4 & -56.3 & -56.3 & -55.7 & -55.4 & -56.6 & -80.9 \\
$3 s$ & -6.9 & -6.8 & -6.9 & -7.9 & -7.4 & -7.5 & -10.1 \\
\hline
\end{tabular}

Notes. The muon is located in $n s$ state.

The obtained energy eigenvalues and wave functions for atomic electrons are used to calculate the energy shifts and relative transition rates of electronic $\mathrm{K} \mathrm{x}$-rays in muonic atoms for various muonic states.

\section{Theoretical model}

The energies and radiative transition rates of electronic $\mathrm{K}_{\alpha}$-x-ray lines were calculated for light elements by treating the muon-electron system self-consistently, as in our previous work [14]. The energy eigenvalues and wave functions of atomic electrons and the muon were obtained with the nonrelativistic HFS method [13]. The validity of the application of this method to muonic atoms was already tested by Mann and Rinker [15]. In muonic atoms, the energies of muons depend on the charge distribution inside the nucleus and it is usual to use the Fermi function [7,12]. However, the energy eigenvalues and wave functions for electrons are less sensitive to the shape of nuclear charge distribution and we can use a simple model. The effect of the finite size of the nucleus was taken into consideration by assuming the nucleus as a uniformly charged sphere. Calculations for the x-ray transition rates were performed in the frozen-orbital approximation [16].

As has been described above, the energy shifts and transition rates depend on the vacancy distribution in muonic atoms. During the muon-cascade process the muonic atom is generally found in positively charged states because of the muonic Auger transitions. There have been developed the computer codes to trace muoncascade process $[17,18]$ and applied for the analysis of muonic x-rays $[19,20]$.

For heavy muonic atoms Vogel [11] and Schneuwly and Vogel [5] indicated that the energy shifts of the electronic $\mathrm{K}$ x-rays in solid and metallic targets are mainly influenced by the atomic state of the muon, and the vacancy distribution plays a minor role. This fact suggests that the inner-shell vacancies produced during the muon cascade are immediately refilled by outershell electrons.

Since for light muonic atoms the muonic Auger transition is more favorable than the muonic x-ray emission [21], it is probable that some electronic vacancies survive in the inner shells. Actually Bacher et al. [23] pointed out that in the case of isolated muonic atoms with low- $Z$ elements, such as $\mathrm{Ne}$ and Ar, the vacancies created in gases remain without refill. However, in the present work we are interested in solid targets and assume that the inner-shell vacancies are quickly refilled and only the outermost electron is missing at the moment of $\mathrm{K}$-x-ray emission. We consider the muonic atom in the form of $\mu^{-} \mathrm{M}^{+}$, where $\mathrm{M}$ is the metallic element.

\section{Results and discussion}

The calculated energy eigenvalues of the muonic atom $\mu^{-} \mathrm{Al}^{+}$are shown in Table 1 for various muonic states and compared with those for neutral $\operatorname{Mg}(Z=12)$ and Al $(Z=13)$ atoms. The muon is considered to be located in the $n s$ state, where $n$ is the principal quantum number of the muonic orbital and $s$ means the orbital angular momentum quantum number $l=0$. It can be seen that when $n$ is large the energy eigenvalues for the muonic atom are close to those for the neutral $\mathrm{Al}$ atom. With decreasing $n$, the absolute values of eigenvalues become smaller and eventually are equal to the values for $\mathrm{Mg}$ for $n<5$. This fact means that the muon penetrates into the inside of the electron orbitals and the screening effect due to the muon is complete. It should be noted that the mean radius of the electronic $1 s$ orbital corresponds to the muonic orbital with $n=14$.

In the HFS method the energy eigenvalues have different meaning from other atomic models, such as the HartreeFock method, due to the Slater approximation for the exchange term and the energies in Table 1 do not correspond to the electron binding energies, i.e. the Koopmans' theorem cannot be applied. However, in the present work we are interested in relative values. In this case the HFS method can give good approximation to the energy shift, as shown by Mann and Rinker [15].

The calculated energy shifts $(\Delta E)$ and the relative radiative rates $(\Delta I)$ of $\mathrm{K}_{\alpha}$-x-ray line in muonic atom $\mu^{-} \mathrm{Al}^{+}$are shown in Table 2 for different muonic orbitals. The energy shifts and the radiative rates are expressed with respect to the corresponding values for the neutral $\mathrm{Mg}$ atom, which has nuclear charge $(Z-1)$. For comparison, the values for the neutral $\mathrm{Al}$ atom are also included in the table. The muon is considered to be in the $n s$ and $n p$ states, where $n$ is chosen from 1 to 20 .

The electronic $\mathrm{K}$ x-rays are emitted only when the energy caused by the muonic transition is larger than the K-electron binding energy. Schneuwly and Vogel [5] showed that the K-vacancy production probability during muon cascade becomes practically zero for $n \geq 11$. This fact means that the $\Delta E$ and $\Delta I$ values for $n>10$ are 
Table 2. Energy shifts and relative radiative rates of $\mathrm{K}_{\alpha} \mathrm{X}$-rays in muonic atoms $\mu^{-} \mathrm{Al}^{+}$relative to the $\mathrm{Mg}$ atom.

\begin{tabular}{llc}
\hline Muonic state & Energy shift & Relative rate \\
\cline { 2 - 3 } & $\Delta E(\mathrm{eV})$ & $\Delta I$ \\
\hline $1 s$ & 0 & 1.00 \\
$2 s$ & 0.1 & 1.00 \\
$5 s$ & 5.0 & 1.00 \\
$5 p$ & 4.7 & 1.00 \\
$8 s$ & 25.1 & 1.01 \\
$10 s$ & 49.7 & 1.01 \\
$10 p$ & 48.6 & 1.01 \\
$14 s$ & 123.4 & 1.05 \\
$20 s$ & 214.0 & 1.24 \\
\hline $\mathrm{Al}$ & 230.1 & 1.51 \\
\hline
\end{tabular}

Notes. For comparison, the values for the $\mathrm{Al}$ atom are shown.

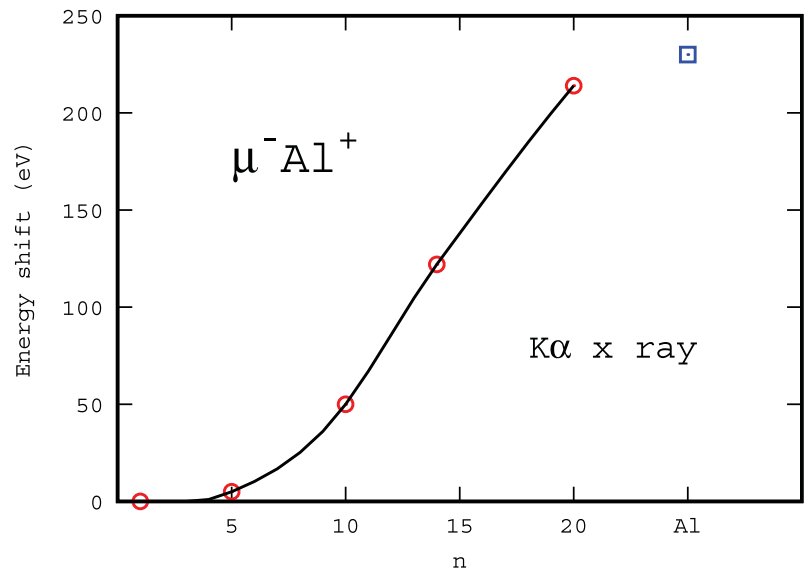

Fig. 1. Energy shift of the $\mathrm{K}_{\alpha} \mathrm{x}$-rays in muonic atom $\mu^{-} \mathrm{Al}^{+}$ relative to the $\mathrm{Mg}$ atom, when the muon is in $n s$ state. The solid line is drawn to guide the eyes. The value for the neutral $\mathrm{Al}$ atom is shown by open square.

unrealistic. Even so it is instructive to see how the $\Delta E$ and $\Delta I$ values approach to those for the neutral Al atom with increasing $n$.

It should be noted that the $\Delta E$ value for the neutral $\mathrm{Al}$ atom in the table is in agreement with the experimental value, $233.0 \mathrm{eV}$, which was obtained as the statistically weighted average of the tabulated values of Deslattes et al. [22] for $\mathrm{K}_{\alpha 1}$ and $\mathrm{K}_{\alpha 2}$ x-rays.

In Figure 1 the calculated energy shifts for muon in the $n s$ state are plotted against $n$. It can be seen that the energy shift is approaching to the $\mathrm{Al}$ value for large $n$, decreases with decreasing $n$, and reduces to the value for $\mathrm{Mg}$ atom $(\Delta E=0)$ at $n=5$. Figure 2 represents the similar plot of the relative radiative rates as a function of $n$. In the case of the $\mathrm{K}_{\alpha}$-x-ray emission rates $n$ dependence is smaller than that for the energy shift and for $n<10$ the radiative rate is almost same as that for the $\mathrm{Mg}$ atom.

In Table 2, we considered only the case where the muon is located in $l=0$ and $l=1$ states. Since the muon is usually captured into highly-excited state, it is

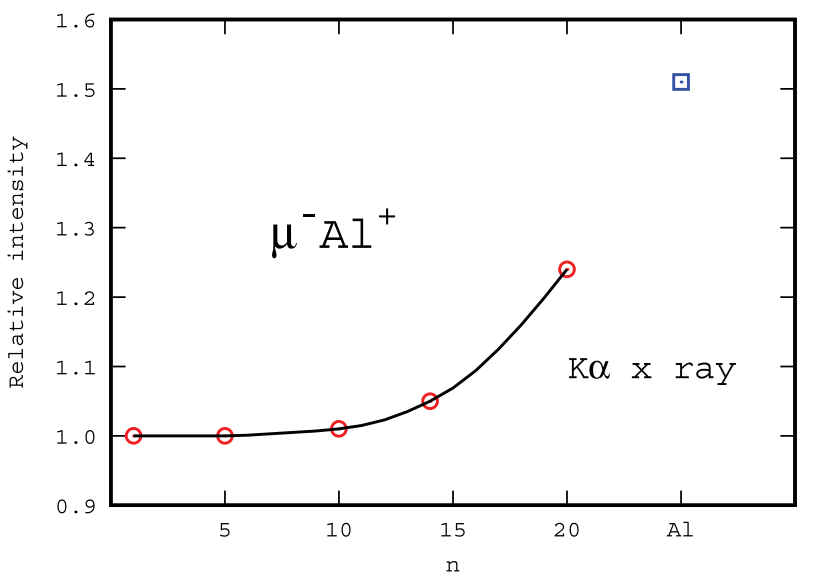

Fig. 2. Relative radiative rate of the $\mathrm{K}_{\alpha} \mathrm{x}$-rays in muonic atom $\mu^{-} \mathrm{Al}^{+}$relative to the $\mathrm{Mg}$ atom, when the muon is in $n s$ state. The solid line is drawn to guide the eyes. The value for the neutral $\mathrm{Al}$ atom is shown by open square.

possible that it still keep higher-l values when the electronic $\mathrm{K}$ x-rays are emitted. Using the muon-cascade program [18] Vogel [11] and Schneuwly and Vogel [5] estimated the electronic K-vacancy production probability for the muonic $n$ state, $P_{K}^{(n)}$. The probability distribution depends on $Z$ and the initial distribution of $l$, and has a peak at $n=7$. This result is obtained for heavy muonic atoms, but we assume that the distribution is similar for light muonic atoms and calculate $l$ dependence of the energy shifts and relative rates. In $\mu^{-} \mathrm{Al}^{+}$we fixed $n$ value to be 7 and calculated the energy shifts and relative transition rates for different $l$ values. The results for $7 l$ muonic states are shown in Table 3. It is clear that $l$ dependence of the energy shift and relative radiative rate is small for light elements, but the former is more sensitive than the latter. It is interesting to note that the energy shift is decreasing with increasing $l$. This behavior is in agreement with the relativistic Dirac-Fock calculations of Rashid and Fricke [12] for muonic Sn atom.

The energy shifts and relative emission rates of $K_{\alpha}$ $\mathrm{x}$-rays in muonic atom $\mu^{-} \mathrm{Fe}^{+}$are given in Table 4 . The energy shifts are given as the energy difference from the corresponding value of the neutral $\mathrm{Mn}$ atom and relative emission rates are expressed as the ratio to the value for $\mathrm{Mn}$. The values for the neutral Fe atom are also listed in the table. The energy shifts for $\mu^{-} \mathrm{Fe}^{+}$are larger than those for $\mu^{-} \mathrm{Al}^{+}$, because the energy difference between the $(Z-1)$ and $Z$ atoms increases with $Z$. However, the energy shifts as a function of $n$ have almost same shape as those for $\mu^{-} \mathrm{Al}^{+}$. On the other hand, the relative emission rates for large $n$ values are smaller in comparison with the case for $\mu^{-} \mathrm{Al}^{+}$. This is due to the smaller $\Delta I$ value for Fe than for $\mathrm{Al}$.

In the present work, we assume that all the innershell vacancies produced during muon cascade are refilled before the electronic $\mathrm{K}_{\alpha}$-x-ray emission. However, it is interesting to study the influence of inner-shell vacancy distribution on energies and radiative transition rates in muonic atoms. The change in energy and emission rate is 
Table 3. $l$ dependence of energy shifts and relative emission rates of $\mathrm{K}_{\alpha}$ x-rays in muonic atom $\mu^{-} \mathrm{Al}^{+}$with $n=7$ relative to the $\mathrm{Mg}$ atom.

\begin{tabular}{lcc}
\hline Muonic state & Energy shift & Relative rate \\
\cline { 2 - 3 } & $\Delta E(\mathrm{eV})$ & $\Delta I$ \\
\hline $7 s$ & 16.1 & 1.00 \\
$7 p$ & 15.6 & 1.00 \\
$7 d$ & 15.0 & 1.00 \\
$7 f$ & 14.8 & 1.00 \\
$7 g$ & 13.6 & 1.00 \\
\hline
\end{tabular}

Table 4. Energy shifts and relative emission rates of $\mathrm{K}_{\alpha} \mathrm{x}$-rays in muonic atoms $\mu^{-} \mathrm{Fe}^{+}$relative to the $\mathrm{Mn}$ atom.

\begin{tabular}{llc}
\hline Muonic state & Energy shift & Relative rate \\
\cline { 2 - 3 } & $\Delta E(\mathrm{eV})$ & $\Delta I$ \\
\hline $1 s$ & 0 & 1.00 \\
$5 s$ & 11.9 & 1.00 \\
$8 s$ & 51.7 & 1.00 \\
$10 s$ & 112.9 & 1.01 \\
$14 s$ & 263.7 & 1.04 \\
$16 s$ & 339.9 & 1.06 \\
$20 s$ & 448.3 & 1.11 \\
\hline $\mathrm{Fe}$ & 491.1 & 1.19 \\
\hline
\end{tabular}

quite sensitive to the number of $2 p$ electrons, because these electrons directly participate in x-ray emission. Table 5 shows the energy shifts and relative emission rates for $\mu^{-} \mathrm{Fe}^{+}$with muon in $7 \mathrm{~s}$ state for different number of vacancies in $2 p$ and $3 p$ shells, which are represented by $N_{2 p}$ and $N_{3 p}$ respectively. It can be seen that the energy shift increases about $30 \mathrm{eV}$ per $2 p$-shell vacancy and increase in energy shift per $3 p$-shell vacancy is about $5 \mathrm{eV}$. The present results can be compared with the theoretical calculations of Rashid and Fricke [12]. They calculated the energy shifts for muonic Sn atom $(Z=50)$ with the muon in $7 i$ state and found that the energy shift increases about $60 \mathrm{eV}$ for each $2 p$ vacancy.

The relative emission rates decrease with increasing $N_{2 p}$ and slightly increase with increasing $N_{3 p}$ for the fixed values of $N_{2 p}$. If we assume that the relative emission rate is proportional to the number of $2 p$ electrons available, the $\Delta I$ value is estimated to be 0.833 for $N_{2 p}=1,0.666$ for $N_{2 p}=2$, and 0.333 for $N_{2 p}=4$. The calculated values in the table are slightly larger than the values obtained with the statistical law.

Most experimental studies on electronic K-x-ray spectra in muonic atoms have been performed for heavy elements $(Z \geq 50)$. However, in the case of pionic atoms Ninomiya et al. [6] measured the energy shift of electronic $\mathrm{K}$ x-rays for $\mathrm{Zn}(Z=30)$. They discussed the difference in electronic K-x-ray energy shifts between pionic and muonic atoms and pointed out importance of electron rearrangement effect in light muonic atoms. Here we neglect such difference and compare their experimental value for pionic $\mathrm{Zn}$ atom with the present model. In Figure 3, the calculated energy shifts of electronic $\mathrm{K}_{\alpha} \mathrm{x}$-rays for muonic atom
Table 5. Energy shifts and relative emission rates of $\mathrm{K}_{\alpha} \mathrm{x}$-rays in muonic atoms $\mu^{-} \mathrm{Fe}^{+}$with muon in $7 s$ state relative to the Mn atom.

\begin{tabular}{lcll}
\hline \multicolumn{2}{l}{ Number of vacancies } & Energy shift & Relative rate \\
\hline$N_{2 p}$ & $N_{3 p}$ & $\Delta E(\mathrm{eV})$ & $\Delta I$ \\
\hline 0 & 0 & 30.2 & 1.00 \\
& 2 & 36.6 & 1.01 \\
& 4 & 43.1 & 1.01 \\
& 0 & 65.1 & 0.869 \\
& 2 & 69.5 & 0.873 \\
2 & 4 & 74.8 & 0.877 \\
& 0 & 90.3 & 0.720 \\
4 & 2 & 95.2 & 0.723 \\
& 4 & 101.1 & 0.727 \\
& 0 & 151.9 & 0.387 \\
& 2 & 158.4 & 0.389 \\
& 4 & 167.3 & 0.393 \\
\hline
\end{tabular}

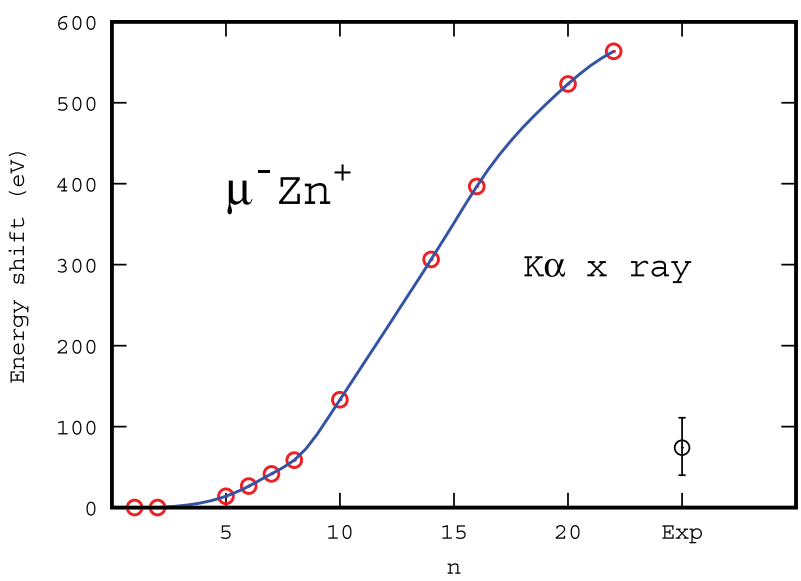

Fig. 3. Energy shift of the $\mathrm{K} \alpha$ x-rays in muonic atom $\mu^{-} \mathrm{Zn}^{+}$ relative to the $\mathrm{Cu}$ atom, when the muon is in $n s$ state. The experimental value for pionic atom is taken from reference [6] and shown by open circle with error bar.

$\mu^{-} \mathrm{Zn}^{+}(Z=30)$ with muon in $n s$ state are compared with the experimental value for the pionic atom [6]. The energy shifts are expressed as the difference from the neutral $\mathrm{Cu}$ atom. It can be said from the figure that the electronic $\mathrm{K}$ $\mathrm{x}$-rays are emitted when the muon is located in the state with $n=8$.

Although this conclusion is based on the assumption that there is no additional vacancy in the $2 p$ shell, it is consistent with the prediction that the probability distribution for $n$, $P_{K}^{(n)}$, has a peak around $n=7$. However, in order to estimate more accurate theoretical energy shifts, we have to calculate the energy shift as the weighted average of the energy shifts with different muonic states by the use of $P_{K}^{(n)}$, which is obtained from the muon-cascade program $[5,11]$.

On the other hand, from their experimental data for heavy muonic atoms and the calculated results of Rashid and Fricke [12], Ninomiya et al. [6] suggested existence of about two $2 p$ vacancies in light muonic atoms when electronic $\mathrm{K}$ x-rays are emitted. It is interesting to note that if we calculate the energy shift for muonic $\mathrm{Zn}$ atom 
with two $2 p$ vacancies in the manner similar to Table 5 , we obtain an additional shift of about $70 \mathrm{eV}$. When we add this additional shift to the $\Delta E$ value at $n=8$, the theoretical value is too large.

It is possible to choose another combination of $n$ and $N_{2 p}$. If we assume that the muon is located in the state $n<5$ and there are two $2 p$ vacancies, i.e. $N_{2 p}=2$, we obtain the $\Delta E$ value in agreement with the experimental data. However, in order to discuss the effect of vacancy distribution on the energy shifts in detail, more systematic experimental and theoretical studies are needed for light muonic atoms.

\section{Conclusion}

The energy shifts and relative radiative rates of electronic $\mathrm{K}_{\alpha}$ x-rays were calculated for light muonic atoms with various states of the muon. The present calculations are based on some simplified assumptions, but $n$ dependence of the muonic orbital on the energy shifts and radiative rates was obtained. On the other hand, $l$ dependence was found to be small.

In the present work, we assumed that electron vacancies created during muon cascade are quickly refilled. However, for light muonic atoms this assumption may not be valid. The calculations indicate that the number of $2 p$ electrons at the moment of electronic K-x-ray emission plays an important role in energy shifts and relative radiation rates.

The knowledge of electron vacancy distribution in cascade process is essential to obtain more accurate information from analysis of electronic x-rays for light muonic atoms. For this purpose, further experimental and theoretical studies on light muonic atoms are needed. We hope that the present results can stimulate such works.

Open access funding provided by MTA Institute for Nuclear Research (MTA ATOMKI).

Open Access This is an open access article distributed under the terms of the Creative Commons Attribution License (http://creativecommons.org/licenses/by/4.0/), which permits unrestricted use, distribution, and reproduction in any medium, provided the original work is properly cited.

\section{References}

1. E.J. Hartmann, R. Bergmann, H. Daniel, H.-J. Pfeiffer, T. von Egidy, W. Wilhelm, Z. Phys. 305, 189 (1982)

2. K. Terada, K. Nishiyama, T. Osawa, S. Tachibana, Y. Miyake, M.K. Kubo, N. Kawamura, W. Higemoto, A. Tsuchimoto, M. Ebihara, M. Uesugi, Sci. Rep. 4, 5072 (2014)

3. R. Arlt, D. Ganzorik, T. Krogulski, H.-G. Ortlepp, S.M. Polikanov, B.M. Sabirov, V.D. Fromm, U. Schmidt, G. Schnefii, R. Engfer, JETP Lett. 20, 291 (1974)

4. W.D. Fromm, Z. Ganzorig, T. Krogulski, H.-G. Ortlepp, S.M. Polikanov, B.M. Sabirov, U. Schmidt, R. Arlt, R. Engfer, H. Schneuwly, Phys. Lett. 55B, 377 (1975)

5. H. Schneuwly, P. Vogel, Phys. Rev. A 22, 2081 (1980)

6. K. Ninomiya, H. Sugiura, T. Nakatsuka, Y. Kasamatsu, H. Kikunaga, W. Sato, T. Yoshimura, H. Matsumura, K. Takamiya, M.K. Kubo, K. Sueki, A. Yokoyama, Y. Hamajima, T. Miura, K. Nishiyama, A. Shinohara, J. Radioanal. Nucl. Chem. 272, 661 (2007)

7. P. Vogel, Phys. Rev. A 7, 63 (1973)

8. P. Vogel, At. Data Nucl. Data Tables 14, 6 (1974)

9. T. von Egidy, J.P. Desclaux, Z. Phys. A 288, 23 (1978)

10. P. Vogel, A. Zehnder, A.L. Carter, M.S. Dixit, E.P. Hincks, D. Kessler, J.S. Wadden, C.K. Hargrove, R.J. McKee, H. Mes, H.L. Andersen, Phys. Rev. A 15, 76 (1977)

11. P. Vogel, Phys. Lett. 58B, 52 (1975)

12. K. Rashid, B. Fricke, Z. Phys. A 304, 193 (1982)

13. F. Herman, S. Skillman, Atomic Structure Calculations (Prentice-Hall, Englewood Cliffs, NJ, 1963)

14. H. Adachi, T. Mukoyama, J. Phys. 48, C9-733 (1987)

15. J.B. Mann, G.A. Rinker, Jr., Phys. Rev. A 11, 385, (1975)

16. S.T. Manson, D.J. Kennedy, At. Data Nucl. Data Tables 14, 111 (1974)

17. J. Hüfner, Z. Phys. 195, 365 (1966)

18. V.R. Akylas, P. Vogel, Comput. Phys. Commun. 15, 291 (1978)

19. F.J. Hartmann, T.V. Egidy, R. Bergmann, M. Kleber, H.J. Pfeiffer, K. Springer, H. Daniel, Phys. Rev. Lett. 37, 331 (1976)

20. P. Vogel, Phys. Rev. A 22, 1600 (1980)

21. G.R. Burbidge, A.H. de Borge, Phys. Rev. 89, 189 (1953)

22. R.D. Deslattes, E.G. Kessler, Jr., P. Indelicato, L. de Billy, E. Lindroth, J. Anton, Rev. Mod. Phys. 75, 35 (2003)

23. R. Bacher, D. Gotta, L.M. Simons, J. Missimer, N.C. Mukhopadhyay, Phys. Rev. Lett. 54, 2087 (1985) 